\title{
Fluorescencia de los Tejidos Queratinosos
}

\author{
Fluorescence in Keratin Tissues
}

Roger L. McMullen; Susan Chen \& David J. Moore

\begin{abstract}
McMULLEN, R. L.; CHEN, S. \& MOORE, D. J. Fluorescencia de los tejidos queratinosos. Int. J. Morphol., 30(3):956-963, 2012.
RESUMEN: Utilizando una técnica de fluorescencia, registramos mapas de los fluoróforos presentes en varios tipos de tejidos queratinosos. Descubrimos que la fluorescencia de todas las muestras analizadas tienen rasgos en común. Observamos el aminoácido triptófano en todos los tejidos. Asimismo, nos parece que la máxima principal es debido a las kinureninas que son productos metabólicos y degradativos del triptófano. Esta máxima constituye un conglomerado de varias moléculas como L-kinurenina, 3-hidroxikinurenina y $\mathrm{N}$-formilkinurenina. La proporción de kinureninas que tiene el triptófano depende del tipo de tejido y del grado de pigmentación. También, la longitud de onda de excitación y emisión depende de estos parámetros.
\end{abstract}

PALABRAS ClAVE: Tejidos queratinosos; Cabello; Piel; Fluorescencia; Uña; Pezuña; Caparazón; Cuerno.

\section{INTRODUCCIÓN}

Los tejidos queratinosos contienen muchos cromóforos que dan fluorescencia en las regiones de radiación visible y ultravioleta. Generalmente, las queratinas se clasifican como suaves o duras. Este aspecto se determina por la composición física y química del tejido. Ejemplos de queratinas duras son el pelo, la uña, la garra, la lana, la pezuña y la pluma. Por otro lado, tenemos queratinas suaves como el estrato córneo de la piel donde hay células diferenciadas que contienen filamentos de queratina y no de los organelos metabólicos de las células normales. Estas células diferenciadas se llaman corneocitos y están situadas en una matriz de lípidos que constituye todo el estrato córneo.

Se han hecho varios estudios para investigar los fluoróforos de la piel. Por ejemplo, hay una emisión de fluorescencia bastante fuerte de la dermis debido a los eslabones de distintos tipos de colágeno (Kollias et al., 1998; Gillies et al., 2000; Kollias et al., 2002; Katika \& Pilon, 2006). La emisión de fluorescencia de la dermis es más fuerte que la de la epidermis y es posible distinguir el contraste entre los dos estratos cuando se separan. El fluoróforo principal de la epidermis es el triptófano; aunque también encontramos otro fluoróforo, la kinurenina, que tiene propiedades en común con una molécula de emisión del cabello. Esta molécula tiene las coordenadas del espectro de una longitud de onda de excitación de $380 \mathrm{~nm}$ y de emisión de $450 \mathrm{~nm}$, aproximada- mente (McMullen et al., 2012). Varios estudios demuestran que este fluoróforo proviene de la kinurenina, un producto metabólico del triptófano (Millington, 2006; Daly et al., 2009; Jachowicz \& McMullen, 2011).

Hemos realizado multiples análisis de distintos tipos de cabello (Jachowicz \& McMullen). El factor más influyente de las propiedades de fluorescencia es el grado de pigmentación del cabello. Por ejemplo, descubrimos que la melanina impide la emisión de fluorescencia del triptófano y de la kinurenina. Asimismo, tanto en el cabello como en las uñas encontramos valores idénticos en su longitud de onda de excitación $(378 \mathrm{~nm})$ y de emisión $(452 \mathrm{~nm})$ correspondientes a la kinurenina (McMullen et al.). Por esta razón, nos interesa mucho entender las propiedades de otros tipos de tejidos queratinosos. En este estudio comparamos la fluorescencia de la garra, el cuerno, la uña, la pezuña, el caparazón, la pluma, el cabello y la piel.

\section{MATERIAL Y MÉTODO}

Todos los análisis de fluorescencia fueron realizados utilizando un espectrómetro de fluorescencia (FluoroMax4) fabricado por Horiba Jobin Yvon (Edison, NJ, Estados Unidos). El espectrómetro tiene una sonda de fibra óptica 
que permite el análisis de muestras sólidas. Por consiguiente, pudimos recoger espectros directamente de la superficie de los tejidos. La rapidez de este instrumento nos permite la recolección y el análisis de muchos espectros en poco tiempo $(80 \mathrm{~nm} / \mathrm{s})$. En vez de generar sólo un espectro bidimensional por cada análisis, hicimos la recolección de muchos espectros y registramos un espectro tridimensional que se llama matriz de excitación y emisión. Hicimos una medición de $270 \mathrm{~nm}$ a $450 \mathrm{~nm}$ en incrementos de $2 \mathrm{~nm}$ para medir la excitación. Los espectros de emisión fueron recogidos 15 nm más largos que los de la longitud de onda de excitación que tienen $200 \mathrm{~nm}$ de largo. Por ejemplo, el primer medición de emisión sería de $285 \mathrm{~nm}$ a $485 \mathrm{~nm}$ y el último de $465 \mathrm{~nm}$ a $665 \mathrm{~nm}$.

Hicimos experimentos con muestras de uña, garra, pezuña, caparazón, pluma, cabello y piel. Utilizamos muestras de varias especies de animales. En el caso de la piel examinamos muestras porcinas (Sus scrofa domesticus), caprinas (Capra aegagrus hircus), bovinas (Bos primigenius) y ovinas (Ovis aries) que fueron recogidas de un matadero. Asimismo, llevamos a cabo estudios in vivo con seres humanos, específicamente de la piel (el antebrazo interior) y las uñas. Para hacer una comparación con las uñas, llevamos a cabo estudios de dos pezuñas caprinas procedentes del matadero, una de color negro y otra de blanco, y de una garra de tejón norteamericano (Taxidea taxus) adquirido en Wakeda.com Trading Post (Oakdale, CA, Estados Unidos). Asimismo, hicimos pruebas del caparazón de una tortuga de caja común (Terrapene carolina) y de un armadillo de nueve bandas (Dasypus novemcinctus) adquiridos en Wakeda.com Trading Post (Oakdale, CA, Estados Unidos) y en Jernigan's Taxidermy (Waco, TX, Estados Unidos), respectivamente. Debido a su parecido con la estructura del material de la uña, analizamos muestras de garra, pezuña, caparazón, cuerno bovino (Jernigan's Taxidermy, Waco, TX, Estados Unidos) y cuerno del bisonte americano (Bison bison) (Wakeda.com Trading Post, Oakdale, CA, Estados Unidos). Comparamos el cabello humano con lana ovina (del matadero) y pelo de yak (Bos mutus). Adquirimos cabello de seres humanos de varios tonos y pelo de yak de un distribuidor (International Hair Importers \& Products, Glendale, NY, Estados Unidos).

También llevamos a cabo estudios de varios tipos de pluma. Normalmente, hay muchos cromóforos en las plumas y por esta razón analizamos muestras sin pigmentación. En este informe solamente mostramos los resultados de plumas de cacatúa de moño amarillo (Cacatua galerita) y de paloma bravía (Columba livia) porque no tienen pigmentación. En todos los casos, limpiamos las muestras con una solución de 3\% (peso/peso) de laurilsulfato de sodio antes de los experimentos.

\section{RESULTADOS Y DISCUSIÓN}

En este estudio intentamos identificar los fluoróforos que existen en común entre varios tipos de tejidos queratinosos. Utilizando una técnica que genera las matrices de excitación y emisión, registramos una huella dactilar de todos los fluoróforos analizados. Comenzamos con tejidos parecidos en su morfología, por ejemplo, de garra, de uña y de pezuña que tienen en común muchas propiedades. También analizamos cabello y cuerno porque tienen propiedades físicas y químicas en común con éstos. Todos estos tejidos se conocen como queratinas duras. A diferencia de estas queratinas duras, el estrato córneo de la epidermis se considera una queratina suave. Estructuralmente, la piel contiene este estrato superficial que consiste en células diferenciadas que están situadas en una matriz de lípidos. Estas células que se llaman corneocitos contienen fibras de queratina que se encuentran ordenadas como filamentos intermedios. Igualmente, las queratinas duras contienen alfa queratina, aunque la morfología de cada tipo de tejido es distinta. En el texto siguiente describimos nuestras conclusiones sobre las diferencias en las propiedades fotoquímicas de los tejidos analizados.

El cabello. De todos los tejidos, el que más hemos estudiado es el cabello, utilizando esta técnica de fluorescencia. En estudios previos, descubrimos evidencia de la presencia de triptófano y de kinureninas, específicamente Lkinurenina, 3-hidroxikinurenina y $\mathrm{N}$-formilkinurenina (Millington; Daly et al.; Jachowicz \& McMullen). Hay dos rutas metabólicas procedentes del triptófano: la de la kinurenina y la de la melatonina. En el cabello solamente encontramos evidencia de la presencia de kinurenina y no de melatonina (McMullen et al.). La kinurenina es un producto de degradación del triptófano. Descubrimos que la radiación ultravioleta perjudica el cabello, ya que reduce la cantidad de triptófano y aumenta el nivel de kinurenina. Este fenómeno es confuso porque también las kinureninas están expuestas al daño de los rayos ultravioletas. Por lo tanto, cuando disminuye la cantidad de triptófano, aumentan las kinureninas, ya que son productos de degradación, a la vez que también disminuyen debido al daño que sufren.

Observamos que la cantidad de pigmento en el cabello juega un papel en sus propiedades fotoquímicas. De hecho, la melanina impide que la luz solar llegue a los otros cromóforos. Por esta razón, hay menos emisión de fluorescencia por parte del triptófano y de las kinureninas. Este fenómeno se puede apreciar en la Figura 1, dónde se ve más emisión en el cabello rubio que en el cabello castaño. La concentración de triptófano y kinureninas es más alto en el caso del cabello rubio. Las coordenadas de triptófano en el 
cabello castaño son lex $=290 \mathrm{~nm}$ y lem $=343 \mathrm{~nm}$ y en el cabello rubio, lex $=292 \mathrm{~nm}$ y lem $=337 \mathrm{~nm}$. La diferencia en la cantidad de melanina en las dos muestras exhibe un leve cambio de posición en el espectro al registrar el valor de longitud de onda. También hay más máximas de las kinureninas que son de mayor intensidad en el caso del cabello rubio. La máxima principal de las kinureninas tiene las coordenadas de lex $=366 \mathrm{~nm}$ y lem $=433 \mathrm{~nm}$ en el cabello castaño y lex $=394 \mathrm{~nm}$ y lem $=465 \mathrm{~nm}$ en el cabello rubio. Así pues, encontramos una variación de posición de la máxima cuando hay un cambio del medio ambiente del fluoróforo. Igualmente interesante es la proporción de intensidad de la máxima de la kinurenina con respecto a la máxima del triptófano. En el cabello castaño encontramos una proporción de $0,239 \pm 0,038$ mientras que en el cabello rubio es mayor con $0,022 \pm 0,001$. En otros tipos de cabello, por ejemplo en varios tonos de castaño, rubio y rojo, observamos espectros parecidos a los del cabello castaño y rubio. Sólo hay diferencias entre el índice de intensidad de la máxima principal de las kinureninas y de la máxima del triptófano. Asimismo llevamos a cabo estudios con pelo de otras especies, por ejemplo con lana de oveja y pelo de caballo, de yak y de bisonte. La Tabla I contiene los valores de las coordenadas del espectro de una longitud de onda que corresponde a la excitación y emisión del triptófano y la máxima principal de las kinureninas. Esta tabla registra la proporción de kinureninas en relación al triptófano. En la Figura 2, se ve la matriz de excitación y de emisión de la lana ovina, como un ejemplo de pelo de animal. En este caso la proporción entre el triptófano y las kinureninas es más elevada que en el caso del cabello. Hay que mencionar que la morfología del cabello y de la lana es muy parecida.

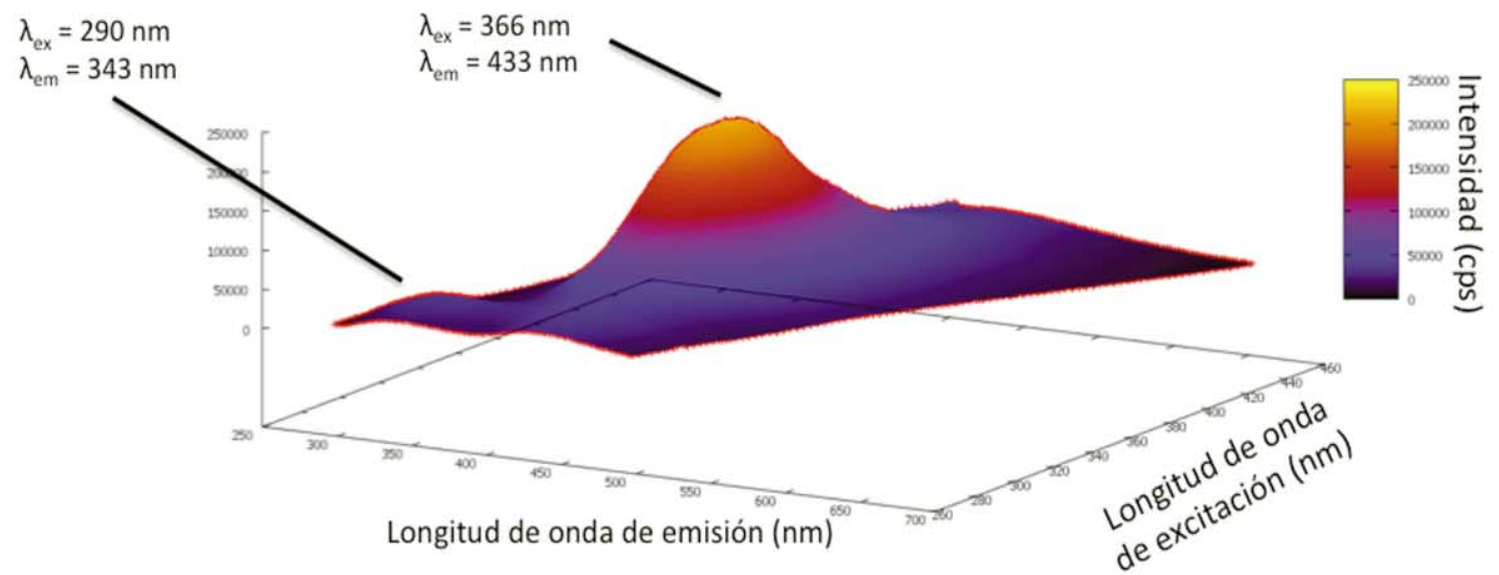

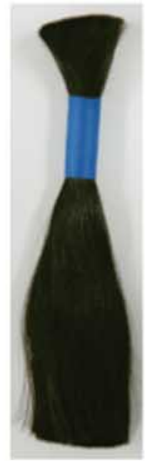

(a)

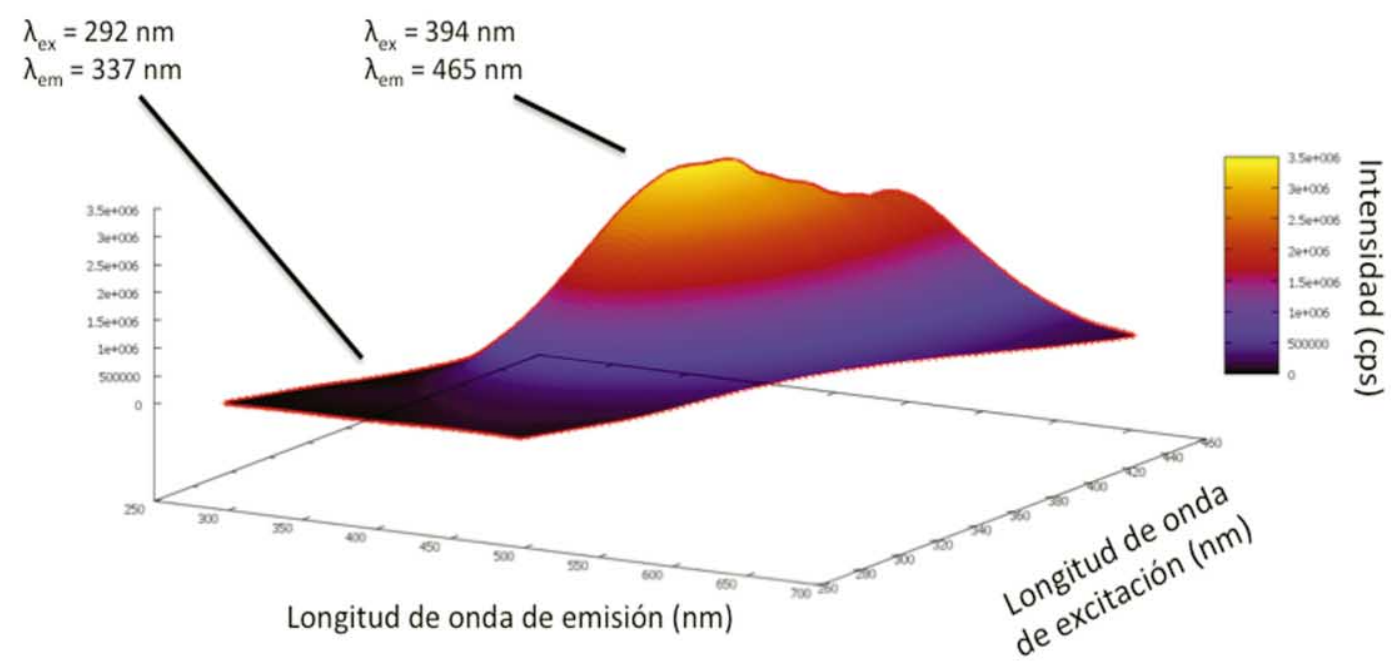

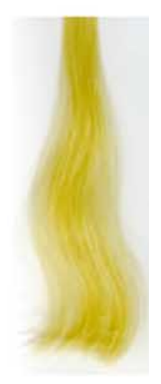

(b)

Fig. 1. Espectros (las matrices) de excitación y de emisión y fotografías (a) de cabello castaño y (b) de cabello rubio. 
La piel. La piel pertenece a la clasificación de queratina suave. Para estudiar la piel es necesario estudiarla in vivo o ex vivo. En estudios in vivo observamos la emisión de fluorescencia del órgano entero de la piel que consiste en la epidermis y la dermis. Por lo tanto, la mayor parte de la emisión proviene de las fibras de colágeno de la dermis. De hecho, la fluorescencia proviene de los eslabones entre las moléculas del colágeno que forman las fibras. Para los objetivos de este informe solamente nos interesaba averiguar el perfil del estrato córneo. Por eso utilizamos una técnica con la enzima tripsina para disgregar el estrato córneo del resto de la piel (Kligman \& Christophers, 1963). El espectro del estrato córneo muestra que hay una máxima principal (con las coordenadas de lex $=290 \mathrm{~nm}$ y lem $=335 \mathrm{~nm}$ ) que representa el triptófano. También hay una máxima me- nor con las coordenadas de lex $=370 \mathrm{~nm}$ y lem $=435 \mathrm{~nm}$. Creemos que esta máxima puede representar la kinurenina o un derivado suyo.

La uña, la garra, la pezuña, el caparazón y el cuerno. Todos estos tejidos tienen en común propiedades mecánicas que les confieren resistencia. Son materias duras que contienen alfa queratina como el cabello y el estrato córneo. También su estructura es rica en cistina, un aminoácido que produce muchos eslabones que confieren fuerza al tejido. Cada uno de estos tejidos tiene una estructura distinta. Sin embargo, los agrupamos aquí porque todos ellos tienen en común una matriz de células en la base de su estructura a partir de la cual crece la parte principal. En el caso de la tortuga, el caparazón tiene una morfología muy diferente,

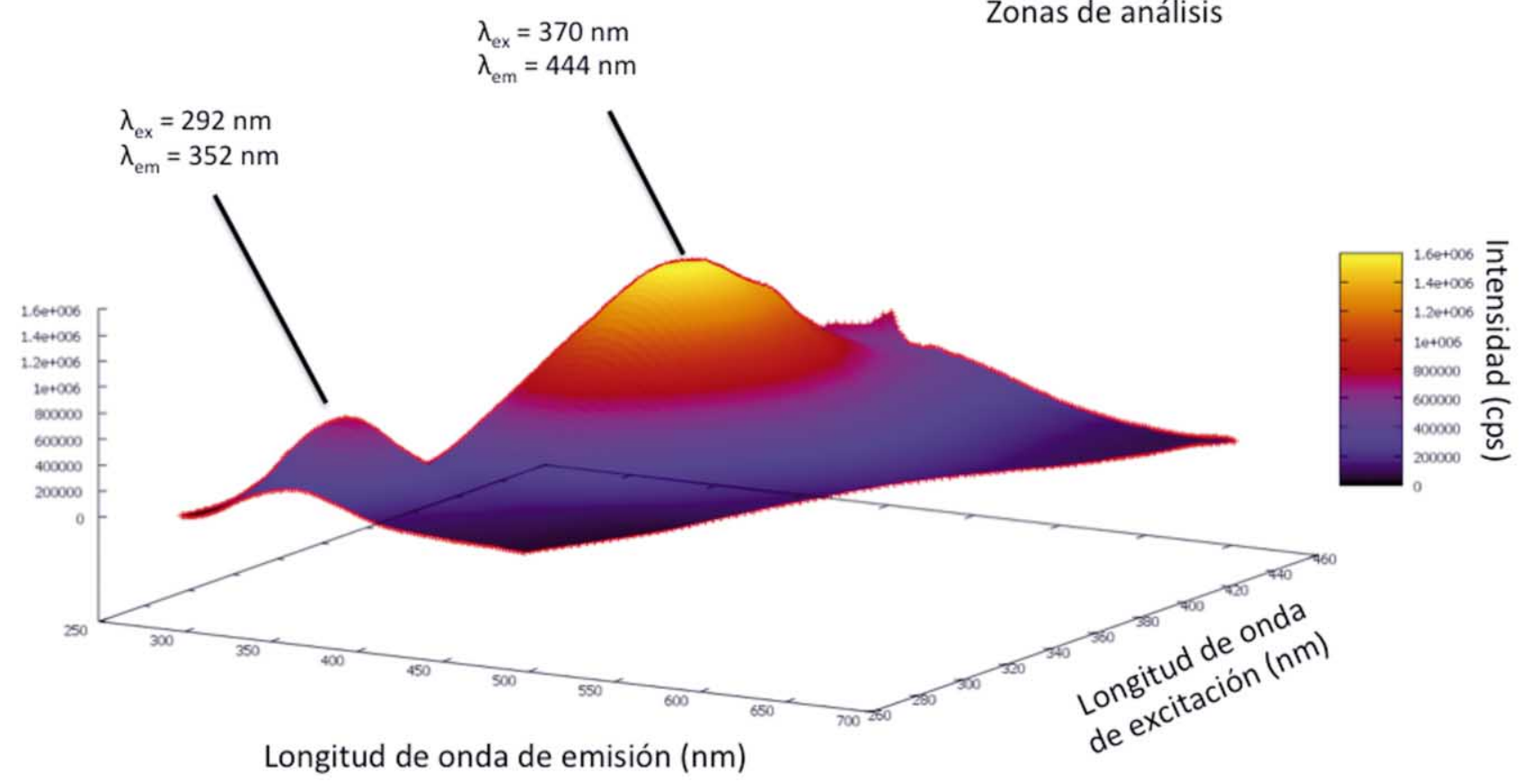

(b)

Fig. 2. (a) Fotografía y (b) espectro de excitación y de emisión de lana ovina. 
Tabla I. Valores de las coordenadas de una longitud de onda de la matriz de excitación y emisión que corresponde a la máxima del triptófano y la máxima principal de las kinureninas.

\begin{tabular}{|c|c|c|c|}
\hline & $\begin{array}{l}\text { Máxima del } \\
\text { triptófano }\end{array}$ & $\begin{array}{l}\text { Máxima de las } \\
\text { kinureninas }\end{array}$ & $\begin{array}{l}\text { Proporción de intensidad } \\
\text { (triptófano/kinureninas) }\end{array}$ \\
\hline \multirow{2}{*}{ Pelo de yak } & $\lambda_{\mathrm{e}_{\mathrm{x}}}=294 \mathrm{~nm}$ & $\lambda_{\mathrm{ex}}=378 \mathrm{~nm}$ & \multirow{2}{*}{$0,093 \pm 0,011$} \\
\hline & $\lambda_{\mathrm{em}}=346 \mathrm{~nm}$ & $\lambda_{\mathrm{em}}=449 \mathrm{~nm}$ & \\
\hline \multirow{2}{*}{ Pelo de bisonte (de la cola) } & $\lambda_{\mathrm{ex}}=290 \mathrm{~nm}$ & $\lambda_{\mathrm{ex}}=358 \mathrm{~nm}$ & \multirow{2}{*}{$0,95 \pm 0,72$} \\
\hline & $\lambda_{\mathrm{e}_{\mathrm{m}}}=334 \mathrm{~nm}$ & $\lambda_{\mathrm{em}}=431 \mathrm{~nm}$ & \\
\hline \multirow{2}{*}{ Pelo de caballo (de la crin) } & $\lambda_{\mathrm{e}_{\mathrm{x}}}=290 \mathrm{~nm}$ & $\lambda_{\mathrm{ex}}=370 \mathrm{~nm}$ & \multirow{2}{*}{$0,16 \pm 0,0043$} \\
\hline & $\lambda_{\mathrm{em}}=341 \mathrm{~nm}$ & $\lambda_{\mathrm{em}}=444 \mathrm{~nm}$ & \\
\hline \multirow{2}{*}{ Lana ovina } & $\lambda_{\mathrm{e}_{\mathrm{x}}}=292 \mathrm{~nm}$ & $\lambda_{\mathrm{ex}}=370 \mathrm{~nm}$ & \multirow{2}{*}{$0,40 \pm 0,070$} \\
\hline & $\lambda_{\mathrm{em}}=352 \mathrm{~nm}$ & $\lambda_{\mathrm{em}}=444 \mathrm{~nm}$ & \\
\hline \multirow{2}{*}{ Pezuña caprina ( negra) } & $\lambda_{\mathrm{ex}}=288 \mathrm{~nm}$ & $\lambda_{\mathrm{ex}}=380 \mathrm{~nm}$ & \multirow{2}{*}{$0,22 \pm 0,018$} \\
\hline & $\lambda_{\mathrm{em}}=338 \mathrm{~nm}$ & $\lambda_{\mathrm{em}}=464 \mathrm{~nm}$ & \\
\hline \multirow{2}{*}{ Pezuña caprina (blanca) } & $\lambda_{\mathrm{e}_{\mathrm{x}}}=292 \mathrm{~nm}$ & $\lambda_{\mathrm{ex}}=350 \mathrm{~nm}$ & \multirow{2}{*}{0,15} \\
\hline & $\lambda_{\mathrm{em}}=378 \mathrm{~nm}$ & $\lambda_{\mathrm{em}}=452 \mathrm{~nm}$ & \\
\hline \multirow{2}{*}{ Garra de tejón norteamericano } & $\lambda_{\mathrm{ex}}=292 \mathrm{~nm}$ & $\lambda_{\mathrm{ex}}=396 \mathrm{~nm}$ & \multirow{2}{*}{0,066} \\
\hline & $\lambda_{\mathrm{em}}=341 \mathrm{~nm}$ & $\lambda_{\mathrm{em}}=465 \mathrm{~nm}$ & \\
\hline \multirow{2}{*}{ Cuerno de bisonte } & $\lambda_{\mathrm{e}_{\mathrm{x}}}=290 \mathrm{~nm}$ & $\lambda_{\mathrm{ex}}=370 \mathrm{~nm}$ & \multirow{2}{*}{$0,19 \pm 0,0039$} \\
\hline & $\lambda_{\mathrm{em}}=341 \mathrm{~nm}$ & $\lambda_{\mathrm{em}}=442 \mathrm{~nm}$ & \\
\hline \multirow{2}{*}{ Cuerno bovino } & $\lambda_{\mathrm{e}_{\mathrm{x}}}=288 \mathrm{~nm}$ & $\lambda_{\mathrm{ex}}=380 \mathrm{~nm}$ & \multirow{2}{*}{$0,027 \pm 0,011$} \\
\hline & $\lambda_{\mathrm{em}}=324 \mathrm{~nm}$ & $\lambda_{\mathrm{em}}=450 \mathrm{~nm}$ & \\
\hline \multirow{2}{*}{ Caparazón de tortuga } & $\lambda_{\mathrm{e}_{\mathrm{x}}}=294 \mathrm{~nm}$ & $\lambda_{\mathrm{ex}}=382 \mathrm{~nm}$ & \multirow{2}{*}{$0,043 \pm 0,025$} \\
\hline & $\lambda_{\mathrm{em}}=345 \mathrm{~nm}$ & $\lambda_{\mathrm{em}}=464 \mathrm{~nm}$ & \\
\hline \multirow{2}{*}{ Caparazón de armadillo } & $\lambda_{\mathrm{e}_{\mathrm{x}}}=290 \mathrm{~nm}$ & $\lambda_{\mathrm{ex}}=368 \mathrm{~nm}$ & \multirow{2}{*}{$0,98 \pm 0,20$} \\
\hline & $\lambda_{\mathrm{em}}=340 \mathrm{~nm}$ & $\lambda_{\mathrm{em}}=445 \mathrm{~nm}$ & \\
\hline
\end{tabular}

porque tiene escudos óseos cubiertos con placas córneas de queratina. La tortuga pertenece a una especie de reptiles que en su anatomía contiene beta queratina en vez de alfa queratina como los mamíferos. Por otra parte, el armadillo es un mamífero, pero como la tortuga también tiene un caparazón de placas óseas cubiertos de queratina. Hay dos tipos de cuernos: cuernos huecos y cuernos fibrosos de queratina. Los cuernos de la mayoría de los animales consisten en un hueso cubierto con una envoltura fina de queratina. Se encuentran en diversas especies, tales como el rinoceronte, el toro y el bisonte. Como un ejemplo de tejido de este grupo, incluimos la figura 3 que contiene un matriz de excitación y emisión para la pezuña caprina. Este espectro tiene propiedades en común con los de otros tejidos queratinos. Hay dos máximas: una de triptofano $(\mathrm{lex}=290 \mathrm{~nm}$ y lem $=345 \mathrm{~nm})$ y otra de kinureninas $($ lex $=378 \mathrm{~nm}$ y lem $=450 \mathrm{~nm})$.

Todos los tejidos que se encuentran en la Tabla I, tienen en común dos propiedades: Hay una máxima que corresponde al triptófano y otra máxima que probablemente corresponde a las kinureninas. Los valores de la longitud de onda de la excitación y emisión dependen del grado de la pigmentación. Como en el caso del pelo, encontramos un cambio de valores que hace que la longitud de onda de la emisión sea más larga. Por ejemplo, la lana ovina y el pelo de yak no tienen pigmentación y por eso la emisión del triptófano es de una longitud de onda más larga que en el caso de pelo de bisonte y de caballo, que tienen el pelo negro. Lo mismo sucede en la pezuña caprina cuando calculamos la cantidad de triptófano. Así, podemos concluir que, mientras la longitud de onda de excitación del triptófano es la misma en cualquier tejido, el valor de emisión cambia según la pigmentación. Por otra parte, la máxima de las kinureninas resulta más difícil de entender. En el caso del cabello humano y del pelo de otros animales, la posición está fijada, más o menos, en una zona entre 360 - $370 \mathrm{~nm}$ para la emisión y 430 - $450 \mathrm{~nm}$ para la excitación. En el caso de las otras muestras, observamos una amplia gama de valores en la máxima principal, entre 350 - $400 \mathrm{~nm}$ en el caso de la excitación y 440 - 465 nm en el caso de la emisión. No obstante, esta máxima probablemente es debida a las kinureninas que se ven muy afectadas por su medio ambiente. 


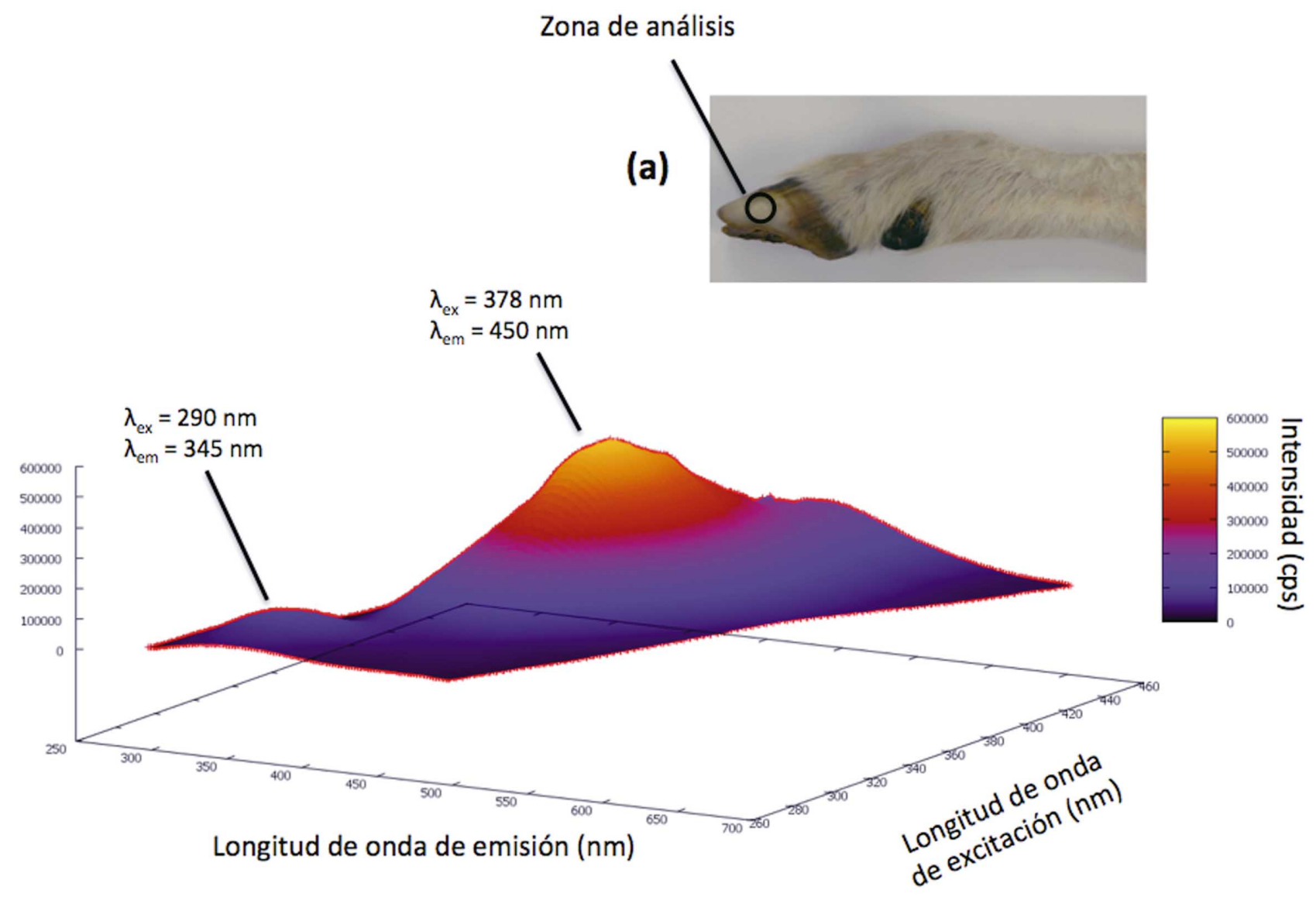

(b)

Fig. 3. (a) Fotografía y (b) espectro de excitación y de emisión de pezuña caprina.

La pluma. La estructura de la pluma consiste en un raquis localizado en el centro con barbas plumáceas a los lados que constituyen una forma parecida a las ramas de un árbol. El punto inferior de la pluma, el cual está en contacto con la piel, se llama cálamo. Como un cabello, la pluma es un apéndice integumentario que se forma a partir de los folículos de la epidermis. A través de estos folículos, los nutrientes pasan por el cálamo y el raquis para alimentar a la pluma. En este estudio examinamos plumas sin pigmentación - plumas blancas. En comparación, tenemos datos de dos tipos distintos de pluma: La de la paloma bravía y la de la cacatúa de moño amarillo. La Figura 4 contiene una imagen de la pluma de la cacatúa donde se ve en el lado izquierdo que tiene un tono amarillo. En este caso, analizamos el lado derecho sin pigmentación y encontramos espectros parecidos a los de los otros tejidos queratinosos, con una máxima principal de coordenadas de lex $=372 \mathrm{~nm}$ y lem $=448 \mathrm{~nm}$ para la paloma y lex $=374 \mathrm{~nm}$ y lem $=441 \mathrm{~nm}$ para la cacatúa (Fig. 4). En ambos casos, el triptófano presenta una máxima de lex $=290 \mathrm{~nm}$ y lem $=330 \mathrm{~nm}$. Cuando observamos la proporción de triptófano en relación a kinureninas calculamos $0,18 \pm 0,010$ y $0,13 \pm 0,023$ para la paloma y para la cacatúa, respectivamente. Estos valores indican que la cantidad de triptófano es menor que en los otros tejidos que contienen más pigmento. Asimismo, observamos que algunos otros tejidos sin pigmentación, como el pelo de yak y la garra, tienen niveles de triptófano más bajos con respecto a la cantidad de kinureninas.

A partir de este estudio es posible encontrar una conexión entre varios tejidos queratinosos de especies diversas. Medimos las cantidades de triptófano y de kinureninas y encontramos que la intensidad y las proporciones de los fluoróforos depende del grado de pigmentación y del tipo de tejido. Curiosamente, todos los tejidos comparten rasgos en común, como es evidente en sus propiedades ópticas y en sus espectros de excitación y emisión.

\section{AGRADECIMIENTOS}

Agradecemos el trabajo que han realizado Núria Diví y Silvia Mendiola Buj con las revisiones del artículo. 
(a)
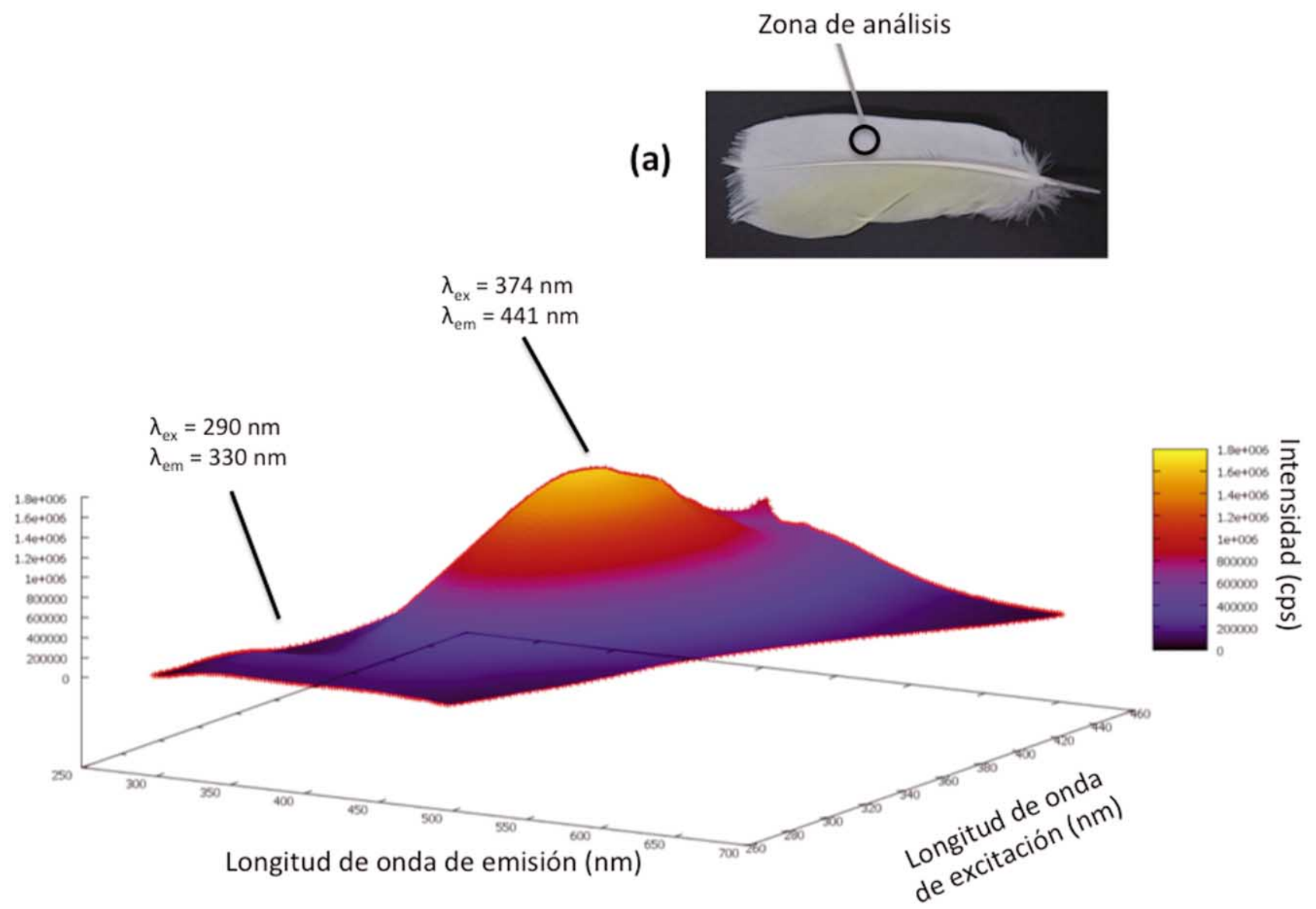

(b)

Fig. 4. (a) Fotografía y (b) espectro de excitación y de emisión de una pluma de cacatúa de moño amarillo.

McMULLEN, R. L.; CHEN, S. \& MOORE, D. J. Fluorescence in keratin tissues. Int. J. Morphol., 30(3):956-963, 2012.

SUMMARY: Utilizing fluorescence, we generated excitation-emission matrices, which provided us with a map of fluorophores present in keratin tissues. Our findings suggest that all of the tested keratin substrates have features in common. We monitor the quantity of tryptophan as well as some of its metabolic and degradation products, the kynurenines. The peak due to kynurenine fluorescence is dominant and is a conglomerate of several molecular species including L-kynurenine, 3-hydroxykynurenine and $\mathrm{N}$-formylkynurenine. The ratio of tryptophan to the kynurenines depends on the type of tissue and the amount of pigmentation present. The emission wavelength also greatly depends on these factors.

KEY WORDS: Keratin tissues; Hair; Skin; Fluorescence; Nail; Hoof; Shell; Horn. 


\section{REFERENCIAS BIBLIOGRÁFICAS}

Daly, S.; Bianchini, R.; Polefka, T.; Jumbelic, L. \& Jachowicz J. Fluorescence and coloration of grey hair. Int. J. Cosmet. Sci., 31(5):347-59, 2009.

Gillies, R.; Zonios, G.; Anderson, R. R. \& Kollias N. Fluorescence excitation spectroscopy provides information about human skin in vivo. J. Invest. Dermatol., 115(4):704-7, 2000.

Jachowicz, J. \& McMullen, R. Factors affecting tryptophan fluorescence in hair. J. Cosmet. Sci., 62:291-304, 2011.

Katika, K. M. \& Pilon, L. Steady-state directional diffuse reflectance and fluorescence of human skin. Appl. Opt., 45(17):4174-83, 2006.

Kligman, A. M. \& Christophers, E. Preparation of isolated sheets of human stratum corneum. Arch. Dermatol., 88:702-5, 1963.

Kollias, N.; Gillies, R.; Moran, M.; Kochevar, I. E. \& Anderson, R. R. Endogenous skin fluorescence includes bands that may serve as quantitative markers of aging and photoaging. J. Invest. Dermatol., 111(5):776-80, 1998.

Kollias, N.; Zonios, G. \& Stamatas, G. N. Fluorescence spectroscopy of skin. Vibr. Spectrosc., 28(1):17-23, 2002.

McMullen, R. L.; Chen, S. \& Moore, D. J. Spectrofluorescence of hair and skin. Int. J. Cosmet. Sci., 34(3):246-56, 2012.

Millington, K. R. Photoyellowing of wool. Part 1: Factors affecting photoyellowing and experimental techniques. Color Technol., 122:169-86, 2006.

\author{
Dirección para correspondencia: \\ Dr. Roger L. McMullen \\ School of Natural Sciences \\ Fairleigh Dickinson University \\ Metropolitan Campus, 1000 River Road \\ Teaneck \\ NJ 07666-1914 \\ UNITED STATE OF AMERICA \\ Email: roger_mcmullen@fdu.edu \\ Recibido : 28-03-2012 \\ Aceptado: 11-06-2012
}

